\author{
L. C. Gómez \\ and F. E. Milioli \\ Departamento de Engenharia Mecânica \\ Escola deEngenharia de São Carlos \\ Av. Trbalhador São Carlense 400, Centro \\ 13566-590 São Carlos, SP. Brazil \\ lubencg@sc.usp.br \\ milioli@sc.usp.br
}

\section{Numerical Simulation of Fluid Flow in CFB Risers - a Turbulence Analysis Approach}

Turbulence parameters are derived from results of numerical simulation of gas-solid flow in circulating fluidized bed (CFB). A two fluid model with constant viscosity is applied considering an Eulerian continuum approach for both phases. An analysis is performed using a direct numerical integration of balance equations without an explicit use of any turbulence model for both phases. Even though velocity fluctuations of lower scales are eliminated by the considered computational mesh, fluctuations of larger scales are detected and analyzed. The results show that the Reynolds stresses behave according to the expected for a turbulent flow, and a reasonable agreement is found regarding literature experiment. Following the literature, granular temperature is derived from the Reynolds stresses, and qualitatively matches the expected behavior as described by the kinetic theory of granular flow. Finally, it is seen that solid's phase turbulence is captured using the present approach.

Keywords. Circulating fluidized beds, hydrodynamics, multiphase flow, riser, turbulence

\section{Introduction}

Turbulence modeling of multiphase flows is quite complex. In addition to the formulation difficulties found in single-phase flows, in multiphase fluids the interface interactions must be described. When the interface is well defined, as in gas-liquid stratified flows, a more rigorous modeling approach can be performed. In gas-solid and other multiphase non-stratified flows, where the interface is not well defined, modeling is much less straightforward.

In order to recover scales of turbulent fluctuations, which are lost in view of numerical mesh scales, models need to artificially incorporate them into the conservative balance equations. Besides, interface interactive terms must be formulated so that the turbulence of a phase is allowed to affect the other phase and vice-versa. Concerning artificially introduced fluctuations, a number of procedures are available for single-phase flows. Those procedures are also applied to multiphase flows, even though modified so that the multiphase physics can be better approached.

In gas-solid flows properties of solid phases have been derived from the so-called kinetic theory of granular flow (KTGF), which is an analogy with the kinetic theory of dense gases (Chapman and Cowling, 1970). Parameters analogous to thermodynamic and physical properties have been derived such as granular temperature and solid phase viscosity. Newtonian rheology has been applied to both phases, and turbulence has been accounted for through artificial procedures like the well known k- $\varepsilon$ model (Peirano and Leckner, 1998).

Many authors consider the KTGF to account for the turbulence of the solid phase in a similar way as the k- $\varepsilon$ model does (Peirano and Leckner, 1998, and Gidaspow, 1994, among others). However, as pointed by Sinclair (1997), this is still a very open question. Sinclair notes that granular temperature is related to velocity fluctuations of individual particles, while the velocity fluctuations of collections of particles relate to turbulent kinetic energy. The concepts are quite distinct and should not be mixed up. It is not possible to determine the thermodynamic temperature from macroscopic continuum hydrodynamic predictions. The same way, it is not fair to determine granular temperature from the mean motion of collections of particles (a continuum defining the solid phase). Granular temperature should be determined either from

Paper accepted March, 2005. Technical Editor: Aristeu da Silveira Neto. velocity fluctuations of particles, or by solving a conservative equation for granular energy.

In this work the turbulence of the solid and gas phases is approached through the direct numerical integration procedure commonly applied in single-phase turbulence analysis. The principle is that, if a computational mesh is sufficiently fine, all hydrodynamic phenomena of a continuum flow field can be resolved through the conventional Navier-Stokes equations. A not fine enough mesh size shall filter turbulent scales of higher frequencies. A relatively coarser mesh shall allow detection of turbulent scales only at lower frequency levels. By applying this approach to the gas and solid phases for a given mesh size, fluctuations of higher frequencies are filtered. However, fluctuations of lower frequencies characterizing large scales of turbulence imposed for instance by geometry, can be predicted. Thereby it is possible to state that at least the large-scale turbulence can be predicted by the usual two fluid model, and no additional procedure is required to deal with turbulence. The way by which the larger scales depend upon smaller scales, and vice-versa, is quite unknown and is a matter for further research. Sundaresan (2000) observes that this relationship mechanism is apparently much different as compared to that observed in turbulent single-phase flows.

Both time and space mesh scales can be further refined regarding those applied in this work. However, caution is required not to undertake the minimum limit on spatial mesh size having in view the validity of the average Eulerian continuum equations for the solid phase. It is not possible to refine the mesh beyond that limit and still maintain the validity of a continuum formulation. Either, for gas and particulate, the limit is of about one order of magnitude above the mean free path among molecules or particles. The limit for the particulate results several orders higher than that for the gas phase. The limit for the gas phase is much lower than that required for catching the smallest turbulent structures, which is about one order above the mean free path among the Kolmogorov dissipative scales.

In this work Reynolds stresses and granular temperature are derived from numerical data. It has been pointed out above that granular temperature should not be determined through continuum hydrodynamic data. In spite of that this is done in this work for two reasons. First, to follow a literature common tendency in view of favorable comparison to experiment (Matonis, Gidaspow and Bahary, 2002). Second, to allow for an evaluation on the validity of such a procedure. Following Peirano and Leckner (1998) and Matonis, Gidaspow and Bahari (2002), granular temperature is assumed equal to two thirds of the turbulent kinetic energy. 


\section{Nomenclature}

$\mathrm{C}_{\mathrm{Ds}}-$ drag coefficient for a single particle in an infinite medium

$\mathrm{d}_{\mathrm{p}}$ - particle diameter, $(\mathrm{m})$

$\mathrm{g}$ - gravity acceleration, $\left(\mathrm{m} / \mathrm{s}^{2}\right)$

$\mathrm{g}_{0}-$ radial distribution function

$\mathrm{G}-$ solid elasticity modulus $\left(\mathrm{N} / \mathrm{m}^{2}\right)$

$\mathrm{k}_{\mathrm{s}}$ - solid phase turbulent kinetic energy by unit of mass $\left(\mathrm{m}^{2} / \mathrm{s}^{2}\right)$

$\mathrm{P}$ - gas pressure $(\mathrm{Pa})$

$\mathrm{Re}_{\mathrm{s}}-$ Reynolds number based on particle diameter

$\mathrm{R}_{\mathrm{g}}$ - ideal gas constant, $(\mathrm{kJ} / \mathrm{kgK})$

$\mathrm{R}_{\min }$ - radius of the minimum spherical volume for continnum validity, (m)

$\mathrm{t}$ - time, (s)

$\mathrm{u}$ - velocity component in radial direction, $(\mathrm{m} / \mathrm{s})$

$\mathrm{v}$ - velocity component in axial direction, $(\mathrm{m} / \mathrm{s})$

$\mathrm{v}_{\mathrm{g}}$ and $\mathrm{v}_{\mathrm{s}}-$ control volume average velocities, $(\mathrm{m} / \mathrm{s})$

$\Delta \mathrm{V}-$ minimum volume for continuum validity $\left(\mathrm{m}^{3}\right)$

$\beta$ - interface drag function, $\left(\mathrm{kg} / \mathrm{m}^{2} \mathrm{~s}\right)$

$\mu$ - dynamic viscosity, $(\mathrm{kg} / \mathrm{ms})$

$\alpha_{\mathrm{g}}$ and $\alpha_{\mathrm{s}}-$ volumetric fractions

$\theta_{\mathrm{s}}$ - solid phase granular temperature $\left(\mathrm{m}^{2} / \mathrm{s}^{2}\right)$

$\rho_{\mathrm{g}}$ and $\rho_{\mathrm{s}}-$ densities, $\left(\mathrm{kg} / \mathrm{m}^{3}\right)$

$\tau_{\mathrm{g}}$ and $\tau_{\mathrm{s}}-$ viscous stress tensors, $(\mathrm{Pa})$

$\phi_{\mathrm{s}}$ - particle sphericity

\section{Subscripts}

(g) and (s) - gas and solid phases

\section{Formulation}

\section{Mathematical Model}

The simulations presented in this work are generated through the hydrodynamic model B developed at IIT (Illinois Institute of Technology) by D. Gidaspow and co-workers. In particular, the MICEFLOW code (Jayaswal, 1991) is utilized. A summary of the governing equations is presented in Table 1. More detailed descriptions can be found in Jayaswal (1991), Gidaspow (1994), Enwald, Peirano and Almstedt (1996) and Cabezas-Gómez and Milioli (2001). The model, generally known as traditional two fluid model, is based on a continuum Eulerian description for each phase. More recently, it has been enhanced by the introduction of the kinetic theory of granular flow (KTGF) (see for instance Gidaspow, 1994).

Table 1. Mathematical Model B (Gidaspow, 1994).

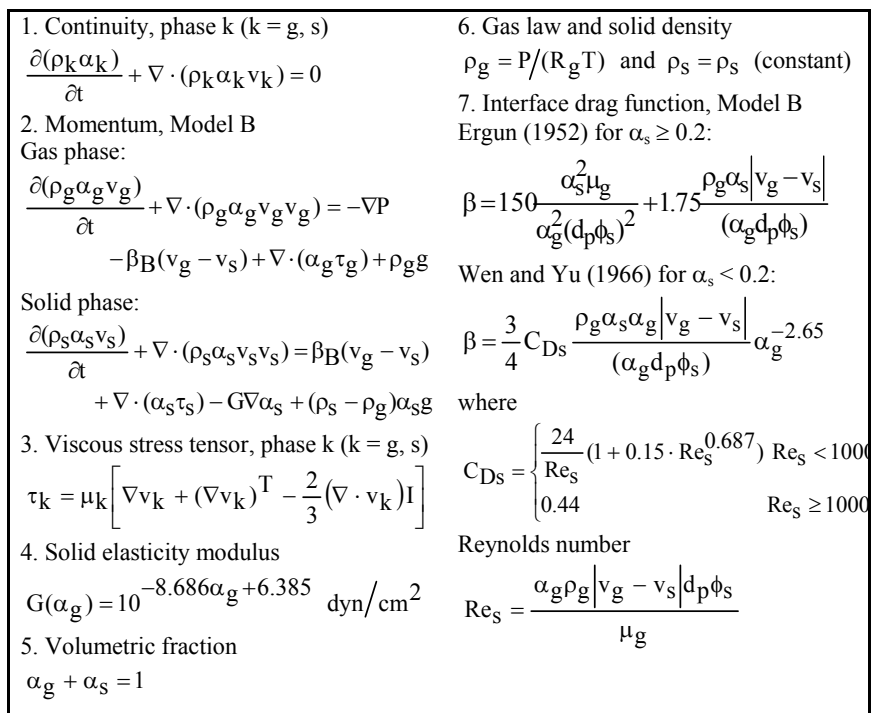

The general model accounts for conservation of mass, momentum and energy for all the phases, and turbulent kinetic energy for solid phases. Newtonian rheology is assumed for all the phases. The computational code allows the description of multiphase flows including two different fluid phases, and several different solid phases, each one characterized by averages of particle diameter, density and shape factor. In this work a gas-solid flow is considered comprising one gas phase (air) and one solid phase (glass beads). Only mass and momentum conservative equations are solved for both phases in two-dimensional flow, and no interface mass transfer is considered. The pressure of the solid phase is modeled by applying the concept of solid's phase elasticity module through the correlation of Rietema and Mutsers (Rietema and Mutsers, 1973, apud Jayaswal, 1991). The interface drag function is modeled through a literature commonly used procedure (Gidaspow, 1994), using Ergun's correlation (Ergun, 1952) when solid's fraction is equal or higher than 0.2 , and Wen and Yu's correlation (Wen and Yu, 1966) when solid's fraction is lower than 0.2.

\section{Derivation of Turbulent and Flow Parameters}

Reynolds stresses are defined by products of time averaged velocity fluctuations (Tennekes and Lumley, 1977). For both phases, normal and shear Reynolds stresses are determined by the following (Mudde et al., 1997, Pan, Dudukovic and Chang, 2000, Matonis, Gidaspow and Bahari, 2002)

$$
\begin{gathered}
\left\langle\mathrm{u}_{\mathrm{k}}^{\prime} \mathrm{u}_{\mathrm{k}}^{\prime}\right\rangle=\frac{1}{\mathrm{~N}} \sum_{\mathrm{n}=1}^{\mathrm{N}} \mathrm{u}_{\mathrm{k}} \mathrm{u}_{\mathrm{k}}-\overline{\mathrm{u}}_{\mathrm{k}}^{2} \\
\left\langle\mathrm{v}_{\mathrm{k}}^{\prime} \mathrm{v}_{\mathrm{k}}^{\prime}\right\rangle=\frac{1}{\mathrm{~N}} \sum_{\mathrm{n}=1}^{\mathrm{N}} \mathrm{v}_{\mathrm{k}} \mathrm{v}_{\mathrm{k}}-\overline{\mathrm{v}}_{\mathrm{k}}^{2} \\
\left\langle\mathrm{u}_{\mathrm{k}}^{\prime} \mathrm{v}_{\mathrm{k}}^{\prime}\right\rangle=\frac{1}{\mathrm{~N}} \sum_{\mathrm{n}=1}^{\mathrm{N}}\left[\left(\mathrm{u}_{\mathrm{k}}-\overline{\mathrm{u}}_{\mathrm{k}}\right)\left(\mathrm{v}_{\mathrm{k}}-\overline{\mathrm{v}}_{\mathrm{k}}\right)\right]
\end{gathered}
$$

where $\mathrm{N}$ represents the number of vectors considered in the time averaged calculations. The considered time interval was 80 seconds of real flow, with a numerical time steps of 0.01 seconds, resulting $\mathrm{N}$ equals to 8000 vectors. The time interval was counted from 20 to 100 seconds, since a statistically developed regime was identified starting at about 20 seconds of real flow.

The solid phase granular temperature was determined as a function of the turbulent kinetic energy by the following (Peirano and Leckner, 1998, Matonis, Gidaspow and Bahari, 2002)

$$
\left.\theta_{\mathrm{s}}=\frac{1}{3}\left(<\mathrm{u}_{\mathrm{s}}^{\prime} \mathrm{u}_{\mathrm{s}}^{\prime}>+<\mathrm{v}_{\mathrm{s}}^{\prime} \mathrm{v}_{\mathrm{s}}^{\prime}\right\rangle\right)=\frac{2}{3} \mathrm{k}_{\mathrm{s}}
$$

where $\mathrm{k}_{\mathrm{s}}$ is the turbulent kinetic energy by unit of mass of the solid phase $\left(\mathrm{m}^{2} / \mathrm{s}^{2}\right)$.

The dynamic viscosity of the solid phase was determined as a function of the granular temperature by the following (Gidaspow, 1994)

$$
\mu_{\mathrm{s}}=\frac{5 \rho_{\mathrm{s}} \mathrm{d}_{\mathrm{p}} \sqrt{\pi \theta_{\mathrm{s}}}}{48(1+\mathrm{e}) \mathrm{g}_{0}}\left[1+\frac{4}{5}(1+\mathrm{e}) \mathrm{g}_{0} \alpha_{\mathrm{s}}\right]^{2}+\frac{4}{5} \alpha_{\mathrm{s}}^{2} \rho_{\mathrm{s}} \mathrm{d}_{\mathrm{p}} \mathrm{g}_{0}(1+\mathrm{e}) \sqrt{\frac{\theta_{\mathrm{s}}}{\pi}}
$$

where $\mathrm{g}_{0}$ is a radial distribution function associated to particles, determined from Bagnold's correlation (Bagnold, 1954); and $\mathbf{e}$ is the restitution coefficient between particles of the solid phase, assumed equal to 0.995 (Gidaspow, 1994). 


\section{Geometry, Initial and Boundary Conditions}

Figure 1 shows the geometry and domain considered in the simulation. The initial and boundary conditions at entrance and exit for both phases are also presented. At entrance a one-dimensional plug flow is considered. At exit the continuity condition is applied to all variables. At the walls a non-slip condition is assumed for the gas phase axial and radial velocities, and for the solid phase radial velocity. Otherwise, a partial slip condition is applied for the solid phase axial velocity following Ding and Gidaspow (1990). Also, a zero gradient in the wall normal direction is prescribed for the gas phase pressure and volumetric fraction. A cartesian numerical mesh $22 \times 297$ was applied which is uniform by blocks in the axial direction as shown in Figure 1. A constant solid phase viscosity was considered which was obtained by Gidaspow and co-workers (Gidaspow, Tsuo and Luo, 1989, and Tsuo and Gidaspow, 1990) from the experimental data of Luo (1987).

\section{Results and Discussion}

Simulated radial profiles of velocities for both phases and solid's fraction are compared to experimental data. Radial profiles of solid's phase axial velocity are considered in comparison to predictions of single-phase laminar and turbulent flows for evaluating the capability of the model of dealing with solid's phase turbulence. Results of Reynolds stresses are presented and discussed. Owing to the lack of experimental data on CFB, the predicted Reynolds stresses are qualitatively compared to literature predictions and experiment in gas-liquid and gas-liquid-solid flows. The predicted granular temperature and solid's phase dynamic viscosity are compared to literature data derived from predictions and experiment in CFB. A discussion is advanced regarding the validity of the continuum approach for the solid phase, and a brief comment is placed related to numerical diffusion.

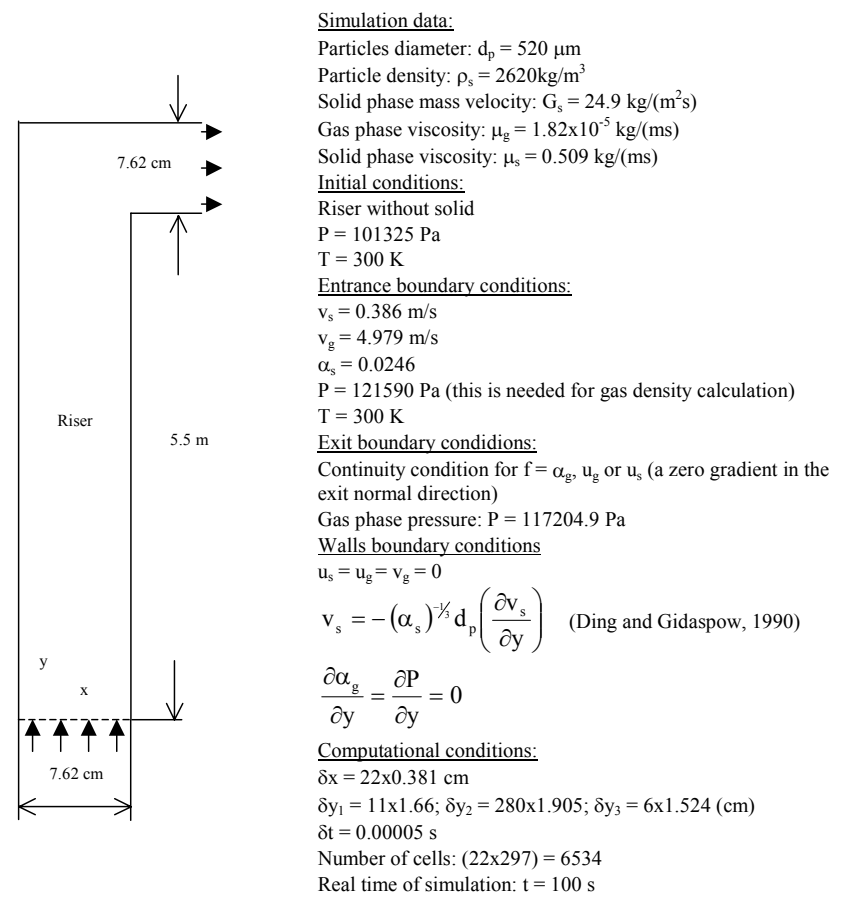

Figure 1. 2D geometry and domain, initial and boundary conditions, and computational conditions for the simulations of the IIT installation (Luo, 1987; Tsuo and Gidaspow, 1990).
On the Flow Pattern, Turbulence and Granular Temperature

Figures 2 and 3 a show time averaged radial profiles of, respectively, axial and radial velocity for both phases. The profiles of radial velocities are almost inversely symmetric around the axis, and show the expected tendency for particle migration towards the walls. The profiles of axial velocities also show the expected annular layer of solid of negative axial velocity along the walls. The velocity profiles unveil the annular plug flow pattern and the segregation of solids towards the walls typical of CFBs. This fact is confirmed by the radial profile of solid fraction presented in Figure $3 \mathrm{~b}$, and by the instantaneous sketches of solid fraction along the column presented in Figure 4. The concentration of solids at the walls is also a cause for clustering, as observed in Figure 4 at the column left wall.


Figure 2. Time averaged radial profiles of axial velocity for both phases $3.4 \mathrm{~m}$ above entrance compared to the experimental data of Luo (1987). Time average from 20 to $100 \mathrm{~s}$. 



Figure 3. Time averaged radial profiles of radial velocity for both phases and solid fraction $3.4 \mathrm{~m}$ above entrance compared to the experimental data of Luo (1987). Time average from 20 to $100 \mathrm{~s}$.



Figure 4. Instantaneous sketches of solid fraction along the column.

Still in Figure 2, the time averaged radial profiles of axial velocity are compared to the experimental data of Luo (1987). For the gas phase the relative deviations are quite significant, mainly at the axis. Otherwise, the axial velocity profile of the solid phase shows a much better behavior. This may be because turbulence is captured by the model for the solid phase but not for the gas phase. In view of the huge difference on the "particle" size of the phases, the applied numerical mesh is able to capture significant scales of turbulence for the solid phase, while severely filters turbulence of the gas phase. A turbulent model for the gas phase seems to be required. The deviations on axial velocity profiles of both phases related to experiment may also be due to experimental uncertainties on local velocities measurements. The better predictions of velocity profiles of the solid phase related to the gas phase are further discussed in Cabezas-Gómez and Milioli (2003).

A more consistent piece of evidence showing that turbulence is in fact being modeled for the solid phase comes from the way that the dynamic viscosity of this phase affects its velocity profiles. Figure 5 shows time averaged radial profiles of solid's phase axial velocity for different values of the solid's phase dynamic viscosity defined around the experimental $0.509 \mathrm{~kg} /(\mathrm{ms})$. It is seen that the velocity profiles become flatter as dynamic viscosity increases. This behavior is consistent with single-phase turbulent flows, as shown in Figure 6 . That figure shows predictions for the single-phase flow of a pseudofluid with properties consistent with the properties of the solid phase in the previous gas-solid flow. Those results were generated with the CFX code for conditions of laminar (Figure 6a) and turbulent (Figure 6b) flows. The same dynamic viscosities of Figure 5 were considered. For the laminar flow the velocity profiles become flatter as the dynamic viscosity is decreased, while for the turbulent flow the contrary happens, i.e. the profiles become flatter as the dynamic viscosity is increased. So, solid's phase turbulence is being modeled by the traditional two fluid model beyond any doubt. At what accuracy, however, is a question that remains to be answered. Of course, the above discussion must be approached with care since it rests on the assumption that the gas-solid two fluid model is accurate. Unfortunately such accuracy is far from established. For instance, Sundaresan (2000) suggests that the 
commonly assumed hypothesis of Newtonian rheology needs revision. The author observes that the so-called sub-grid viscosity is proportional to strain rate in single-phase turbulent flows, while it seems to be inversely proportional to strain rate in gas-solid flows.

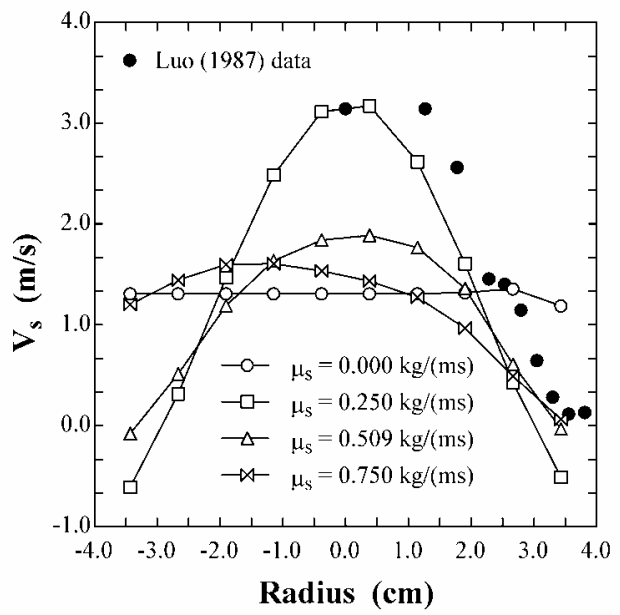

Figure 5. Time averaged radial profiles of solid's phase axial velocity for different solid's phase dynamic viscosities $3.4 \mathrm{~m}$ above entrance compared to the experimental data of Luo (1987) (Cabezas-Gómez and Milioli, 2003).
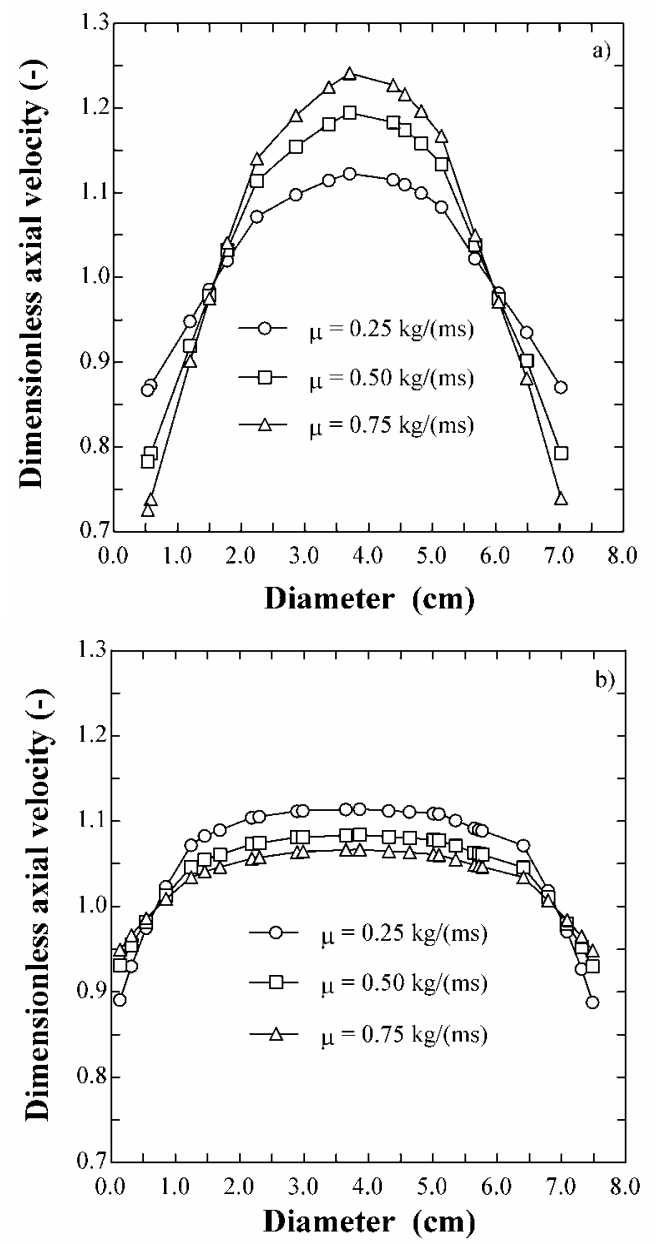

Figure 6. CFX simulated radial profiles of axial velocity for single-phase flows $3.4 \mathrm{~m}$ above entrance for the column of Figure 1, for a pseudofluid with properties consistent with those of the solid phase in the concerning gas-solid flow $\left(\rho=260 \mathrm{~kg} / \mathrm{m}^{3}, \mu=0.25,0.50\right.$ and $\left.0.75 \mathrm{~kg} /(\mathrm{ms})\right)$. (a) For a laminar flow ( $v=3 \mathrm{~m} / \mathrm{s})$; (b) For a turbulent flow $(\mathrm{v}=30 \mathrm{~m} / \mathrm{s})$.
Figures 7 and 8 show radial profiles of normal and shear Reynolds stresses in various different sections of the column. Normal stresses result mostly symmetric and one-modal. Shear stresses result mostly inversely symmetric and two-modal. This behavior is in agreement with observations for gas-liquid columns of bubbles (Mudde et al., 1997, and Pan, Dudukovic and Chang, 2000). The normal axial stresses $\left\langle\mathrm{v}^{\prime} \mathrm{v}^{\prime}\right\rangle$ are superior by three orders of magnitude as compared to the normal radial stresses $\left\langle u^{\prime} u^{\prime}\right\rangle$, and by two orders of magnitude as compared to the shear stresses $\left\langle u^{\prime} v^{\prime}\right\rangle$. This is in disagreement with the results for both gas-liquid bubble columns of Mudde et al. (1997) and gas-liquid-solid bubble columns of Matonis, Gidaspow and Bahari (2002). In those cases normal radial and axial stresses are of the same order, and are one order higher than the shear stresses. However, despite the disagreement regarding order of magnitude, the qualitative behavior of $\left\langle u_{\mathrm{s}}{ }^{\prime} \mathrm{u}_{\mathrm{s}}{ }^{\prime}\right\rangle$ and $\left\langle\mathrm{u}_{\mathrm{s}}{ }^{\prime} \mathrm{v}_{\mathrm{s}}{ }^{\prime}\right\rangle$ is similar to that of the gas-liquid and gas-liquid-solid systems. The normal stresses $\left\langle\mathrm{u}_{\mathrm{s}}{ }^{\prime} \mathrm{u}_{\mathrm{s}}{ }^{\prime}\right\rangle$ in the bubble columns are maximum close to the axis, since radial velocities are maximum at this region owing to a spinning motion of bubble streams. Supposingly, the up flow around the axis is quite uniform and consequently characterized by low fluctuations of radial velocity (Mudde et al., 1997). For the concerning gas-solid flow the profiles of axial and radial velocities cause segregation of solids at the walls and impose higher normal radial stresses around the axis.
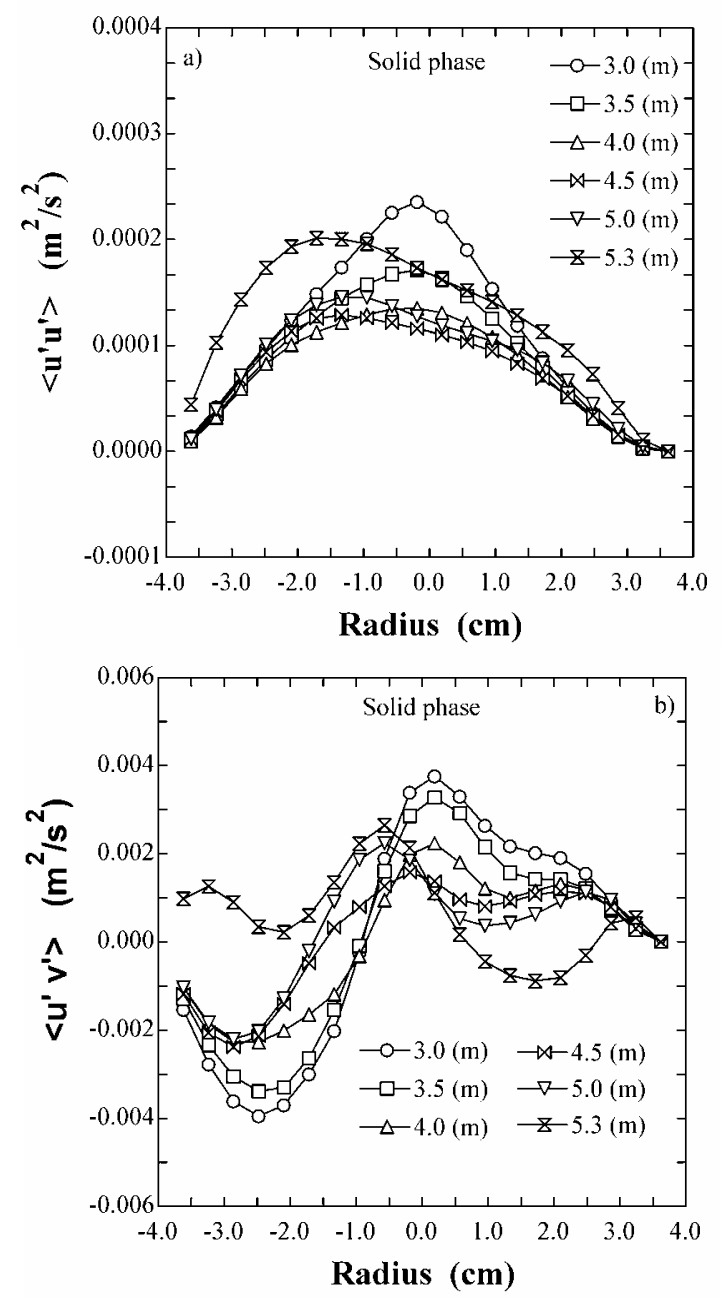

Figure 7. Radial profiles of normal $\left\langle u_{s}{ }^{\prime} u_{s}{ }^{\prime}\right\rangle$ and shear $\left\langle u_{s}{ }^{\prime} v_{s}{ }^{\prime}\right\rangle$ Reynolds stresses in various different sections of the column. 

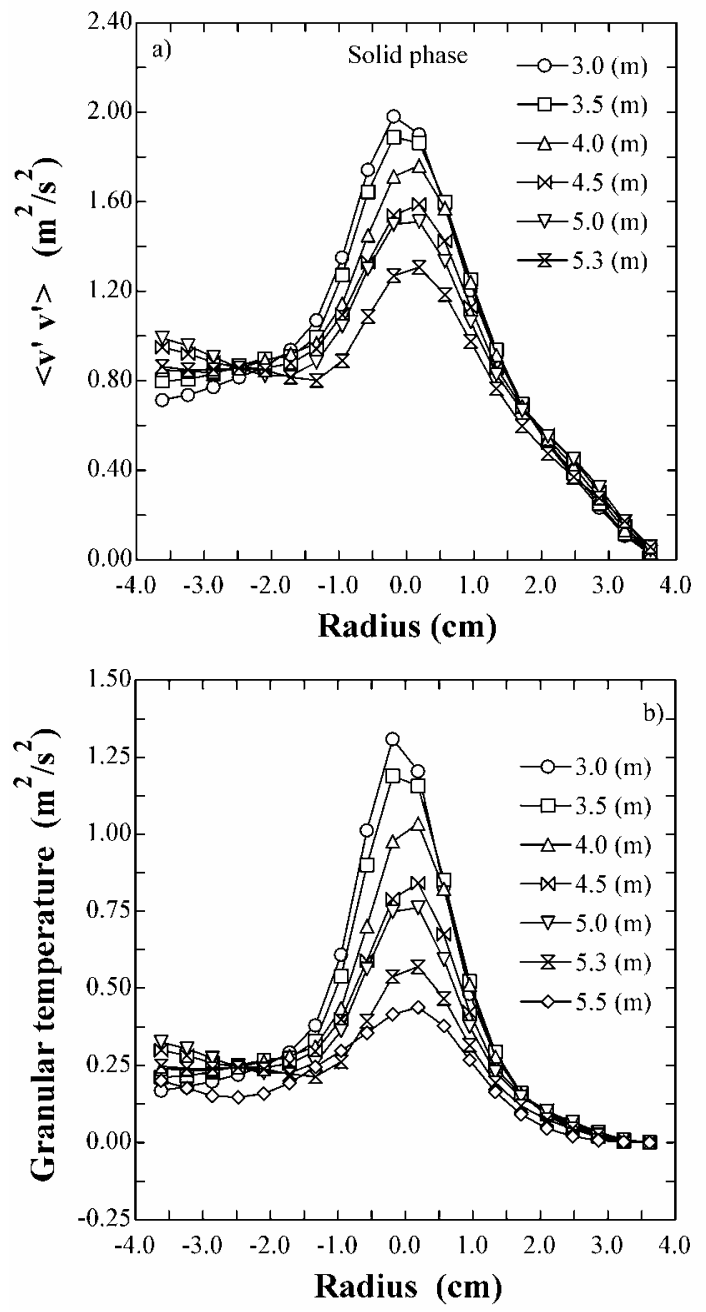

Figure 8. Radial profiles of normal $\left\langle v_{s}{ }^{\prime} v_{s}{ }^{\prime}\right\rangle$ Reynolds stresses and granular temperature in various different sections of the column.

The shear stresses for the concerning gas-solid flow behaves similarly to that of bubble flows. Besides, just like in the bubble columns, the shear stresses for the gas-solid flow tend to zero in regions close to the walls. The major qualitative differences between the considered cases relate to the $\left\langle\mathrm{v}_{\mathrm{s}}{ }^{\prime} \mathrm{v}_{\mathrm{s}}{ }^{\prime}\right\rangle$ profiles. While in the bubble columns of Mudde et al. (1997), Pan, Dudukovic and Chang (2000) and Matonis, Gidaspow and Bahari (2002) a maximum appears close to the walls, in the concerning gas-solid flow a maximum is observed at the axis. In fact this behavior is typical of the $\left\langle u_{s}{ }^{\prime} u_{s}{ }^{\prime}\right\rangle$ profiles observed in the bubble columns. Mudde et al. and Matonis et al. attribute the behavior of the bubbly flows to the development of eddies with scales close to the diameter of the column, while according to Pan et al. it is due to the occurrence of a liquid downflow close to the walls.

In the gas-solid flow, despite the existence of an annular downflow of solids at the walls and a diluted upflow plug around the axis, the normal stresses do not behave like in bubble columns. Despite the mismatching regarding bubble columns, there are evidences supporting the current predictions for gas-solid flow. Gidaspow and Huilin (1998) obtained experimental data on granular temperature in a CFB system. The results show that the axial component of the instantaneous particle velocity standard deviation is up to two orders of magnitude higher than the radial component, meaning that the fluctuations on axial particle velocities in fact determine granular temperature. Such effect is clearly seen in Figure
8 , where the profiles of granular temperature result very similar to the profiles of $\left\langle\mathrm{v}_{\mathrm{s}}{ }^{\prime} \mathrm{v}_{\mathrm{s}}{ }^{\prime}\right\rangle$. The above allows concluding that the differences in magnitude of $\left\langle\mathrm{V}_{\mathrm{s}}{ }^{\prime} \mathrm{v}_{\mathrm{s}}{ }^{\prime}\right\rangle$ regarding both $\left\langle\mathrm{u}_{\mathrm{s}}{ }^{\prime} \mathrm{u}_{\mathrm{s}}{ }^{\prime}\right\rangle$ and $<\mathrm{u}_{\mathrm{s}}{ }^{\prime} \mathrm{v}_{\mathrm{s}}{ }^{\prime}>$, are qualitatively correct.

Figure 9 shows time averaged radial profiles of granular temperature and solid fraction in the column's left side. Granular temperature results maximum at the axis, where the solid fraction is minimum as expected from Gidaspow's results (Gidaspow, 2000). At the central core the flow is diluted, and the mean free path between particles results high. As a consequence, the fluctuations in the motion of particles are increased and granular temperature grows. This behavior is physically coherent reinforcing the correctness of the predictions of normal axial stresses.

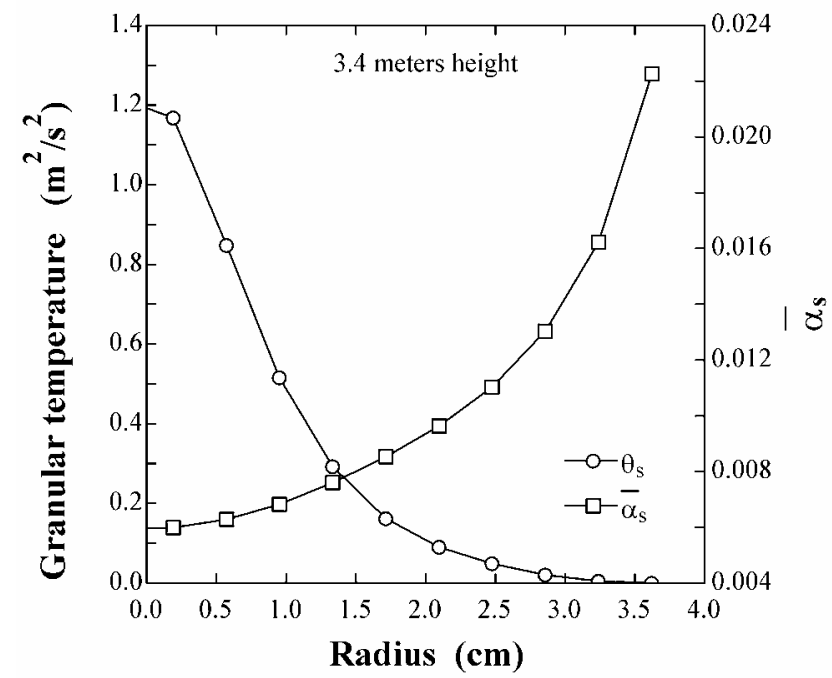

Figure 9. Time averaged radial profiles of granular temperature and solid fraction in the column's left side $3.4 \mathrm{~m}$ above entrance.

Neri and Gidaspow (2000) determined granular temperature by solving a granular energy equation coupled with the momentum equations. Neri and Gidaspow's plots of granular temperature against solids fraction are qualitatively similar to those obtained in this work, which are shown in Figure 10. Still, both the results are qualitatively similar to the figures of Gidaspow and Huilin (1998), who determined granular temperature through experiment. It seems that the same qualitative correct results of granular temperature are found by either solving a granular energy equation or deriving Reynolds stresses. However, despite the agreement in terms of qualitative behavior, the quantitative results are in significant disagreement. Figure 11 presents results for the dynamic viscosity of the solid phase determined as a function of the granular temperature obtained from Reynolds stresses (Equation 5). The average value of solids viscosity determined through Equation 5 resulted about $7.5 \times 10^{-5} \mathrm{~kg} /(\mathrm{ms})$, several orders higher than the experimental average of $0.509 \mathrm{~kg} /(\mathrm{ms})$ used in this work. Such discrepancy is clearly a major consequence of the quantitative deviation on granular temperature. However, it is advisable not to forget that the simulation was performed imposing a constant solid viscosity in each computational cell, disregarding its obvious relation to the solids fraction, which changes dramatically in time and all over the computational domain. The above are clearly matters requiring further thought. 

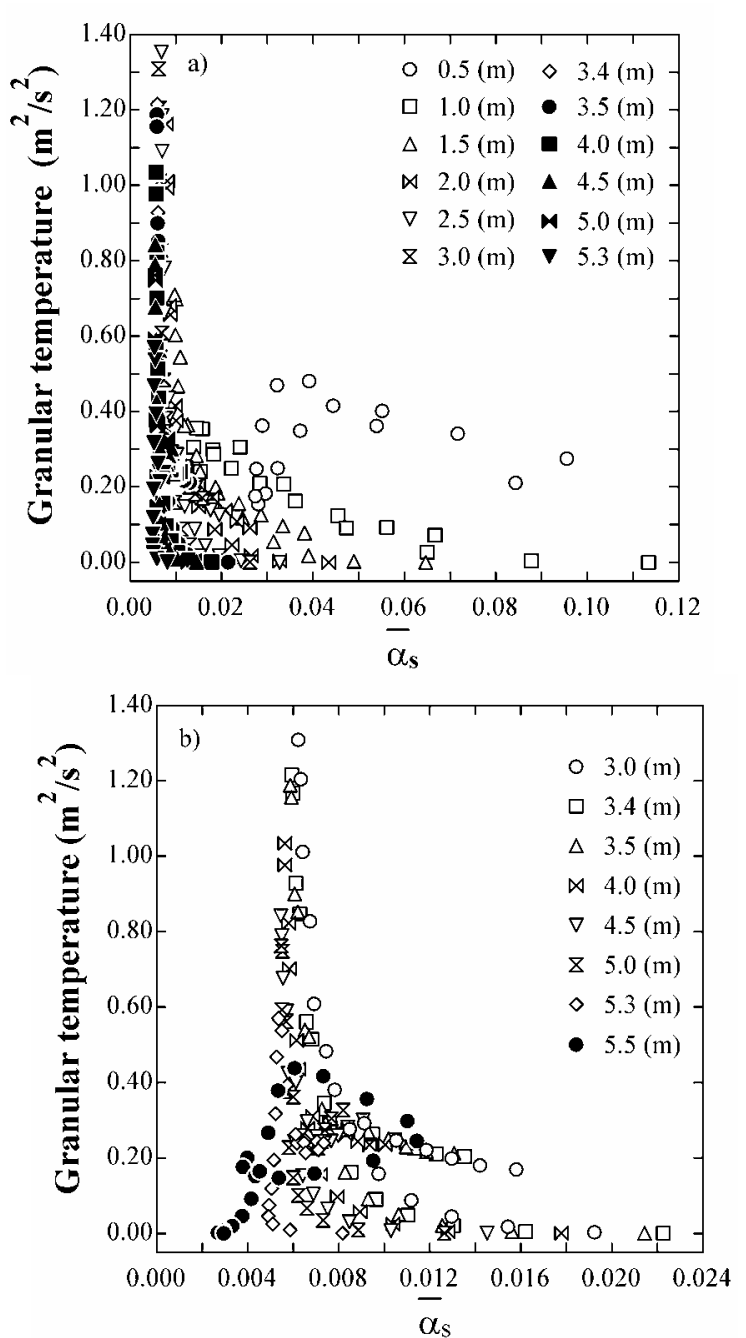

Figure 10. Granular temperature determined from Equation 4 against time averaged solids fraction in various different sections of the column (figures a and $b$ show the same data for different scales of $\bar{\alpha}_{s}$ ).

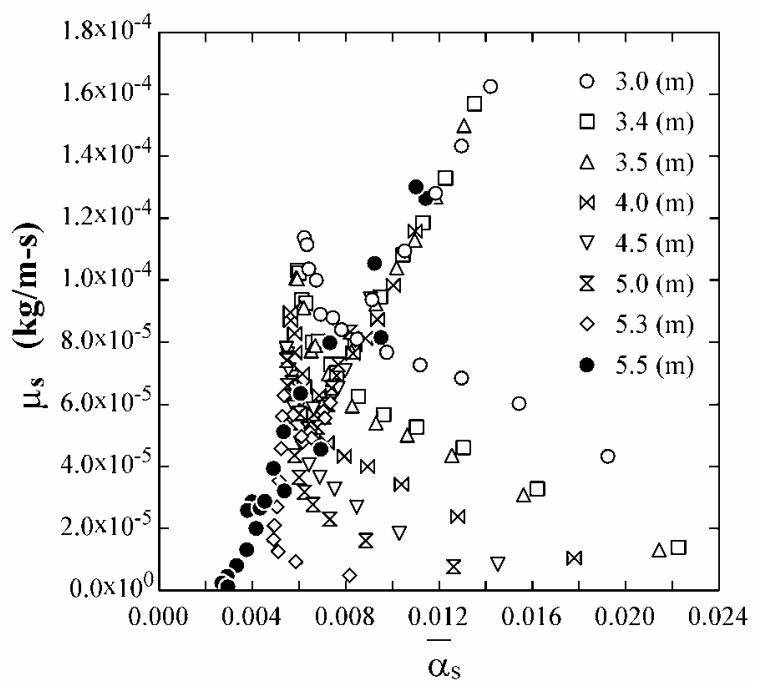

Figure 11. Dynamic viscosity of the solid phase (Equation 5) against time averaged solids fraction.

\section{On the Continuum Assumption}

Celmiņš (1988) proposed a quantitative criterion for the minimum volume on which the volume averaged Eulerian continuum equations for the solid phase are valid. From Celminšs' developments, Fan and Zhu (1998) proposed the following correlation

$$
\frac{\mathrm{R}_{\min }}{\mathrm{d}_{\mathrm{p}}}=\frac{\alpha_{\mathrm{s}}^{-\frac{1}{3}}}{\sqrt{2 \delta}}\left(1-\alpha_{\mathrm{s}}\right)
$$

This expression stands for spherical particles uniformly distributed according to a three dimensional hexagonal array. $\mathrm{R}_{\min }$ represents the radius of the minimum spherical volume for which the solid phase behaves as a continuum. $\delta$ is a relative deviation between the asymptotic and the ideal hexahedral particle array for $\alpha_{\mathrm{s}}$ values. The minimum volume is given by

$$
\Delta \mathrm{V}=\frac{4 \pi}{3} \mathrm{R}_{\min }^{3}
$$

Figure 12 shows the minimum volume $\Delta \mathrm{V}$ as a function of solid's fraction for the particle size of the present simulation ( $\delta$ was taken equal to $1 \%$ following Fan and Zhu, 1998). For solid's fraction typical of the present simulation the graph shows a minimum volume in the range of about 3 to $20 \mathrm{~cm}^{3}$. As the present work deals with 2-D Cartesian simulations the face of a cube with the minimum volume is to be considered. It results between 2.1 and $7.4 \mathrm{~cm}^{2}$. The computational cells applied in the present simulation were between 0.58 and $0.73 \mathrm{~cm}^{2}$. Even though the present simulation does not meet the required continuum criterion, several recent published simulations do not comply with the concerning criterion as well. At what extent such violation affects predictions is not known. Anyway, it is strongly advisable the utilization of adaptative mesh procedures through which local instantaneous solid's fractions all over the domain are used to redefine cell sizes so that the continuum condition is always satisfied.

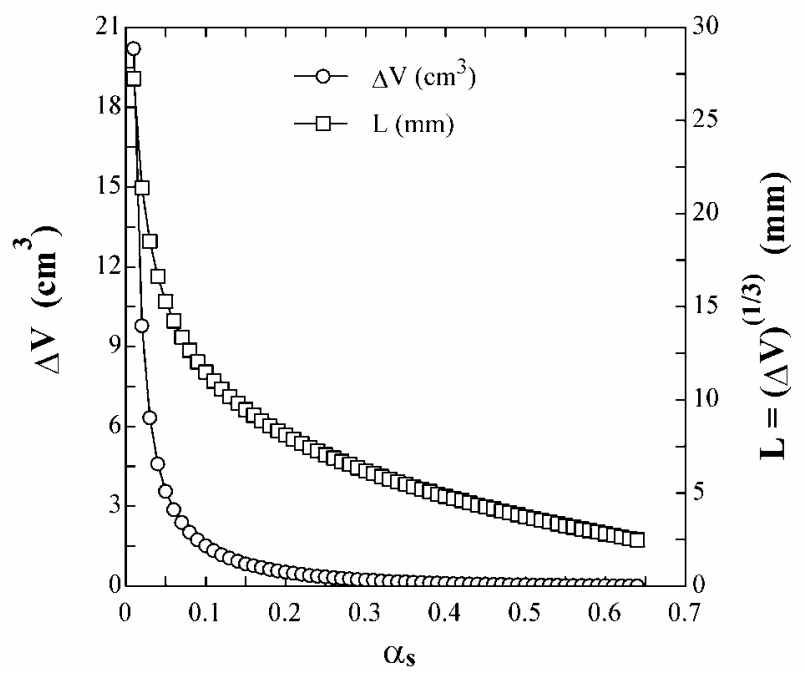

Figure 12. Minimum volume for what the solid phase behaves as a continuum (as determined from Equations (6) and (7), with $d_{p}=0.520 \mathrm{~mm}$ and $\delta=1 \%$ ). 
The computational mesh $22 \times 297$ used in this work is much finer than the $12 \times 75$ mesh used in a previous work for the same conditions (Cabezas-Gómez and Milioli, 2003). Figure 13 shows a comparison of time averaged radial profiles of axial velocities for the two meshes. Despite quantitative differences, the predictions result qualitatively similar. This shows that mesh size has no effect on qualitative predictions behavior, at least for the cases considered. One possible reason for the quantitative disagreement could be numerical diffusion, since an upwind discretization scheme has been applied. However, as seen in Figure 5, the radial profile of solid's phase axial velocity for an inviscid case results quite flat and, therefore, free of "significant" viscous effects whether physical or numerical. This rules out numerical diffusion as a significant effect. The results of Figure 5 stand for the coarser $12 \times 75$ mesh. It may well be that the quantitative disagreement on the predictions for the different meshes is a major consequence of the extent or accuracy at what turbulence is captured. This reinforces the need for working with cell sizes that keep valid the continuum hypothesis on a local instantaneous basis. Suposingly, violations of the continuum shall provide quite inaccurate turbulent information, and thereby quite inaccurate predictions.


Figure 13. Time averaged radial profiles of axial velocity for both phases $3.4 \mathrm{~m}$ above entrance for two different meshes (mesh 1: 12x75; mesh 2: 22x297; • experimental data of Luo (1987)).

\section{Conclusions and Remarks}

The simulations showed that the traditional two fluid model adequately catches the mean macroscopic features of a gas-solid flow in the riser of a CFB. This includes the well-known low frequency flow oscillations, annular plug flow pattern, and clustering. The effect of dynamic viscosity over solid's phase velocity profiles resulted consistent with single-phase turbulent flows instead of laminar flows. It was found that solid's phase turbulence is captured by the traditional two fluid model beyond any doubt. At what accuracy, however, is a question that remains unanswered. Such conclusion must be approached with care since it rests on the assumption that the gas-solid two fluid model is accurate. Unfortunately such accuracy is far from established.

Turbulent parameters were determined assuming a direct numerical integration procedure. Despite quantitative disagreement regarding literature results, both simulated and experimental, the predictions showed a good qualitative behavior for the solid phase. The behavior of normal axial Reynolds stresses was different from that observed in gas-liquid and gas-liquid-solid systems. A dense downflow of solids was observed at the walls leaving a dispersed upflow plug around the axis. The maximum normal axial Reynolds stresses occurred around the axis, and caused the granular temperatures to be higher at this region. Contrary to the bubble columns, normal axial Reynolds stresses resulted orders of magnitude higher than normal radial and shear Reynolds stresses.

It was pointed not to be fair to determine granular temperature from the mean motion of collections of particles the same way that it is not possible to determine the thermodynamic temperature from macroscopic continuum hydrodynamic predictions. However, granular temperature was derived from continuum hydrodynamic data so that such a procedure could be analyzed. Comparisons of granular temperature were performed against both experiment and granular energy predictions. Despite qualitative agreement was achieved, the quantitative results are in significant disagreement. Whether the macroscopic oscillations of the flow can be related to the particle's velocity fluctuations is still an open question. It appears that granular temperature should be determined either from velocity fluctuations of particles, or by solving a conservative equation for granular energy.

A minimum limit should be enforced on numerical spatial mesh sizes having in view the validity of the average Eulerian continuum equations for the solid phase. Theoretically, It is not possible to refine the mesh beyond that limit and still maintain the validity of a continuum formulation. The computational cells applied in the present simulation do not meet the required continuum criterion the same way several recent published simulations do not. At what extent such violation affects predictions is not known. Anyway, it is strongly advisable the utilization of adaptative mesh procedures through which local instantaneous solid's fractions are used to redefine cell sizes so that the continuum condition is satisfied.

It is suggested that quantitative disagreement on predictions for different meshes be a major consequence of the extent or accuracy at what turbulence is captured. This reinforces the need for working with cell sizes that keep valid the continuum hypothesis on a local instantaneous basis. Supposingly, violations of the continuum shall provide quite inaccurate turbulent information, and thereby quite inaccurate predictions.

\section{Acknowledgements}

This work was supported by FAPESP (Fundação de Amparo a Pesquisa do Estado de São Paulo) through doctoral and postdoctoral scholarships for the first author (processes 98/13812-1 and 02/12038-8). 


\section{References}

Bagnol, R.A., 1954, "Experiments on a Gravity-Free Dispersion of Large Solid Spheres in a Newtonian Fluid under Shear", Proceedings of Royal Society, A225, pp. 49-63.

Cabezas-Gómez, L. and Milioli, F.E., 2003, "Numerical Study on the Influence of Various Physical Parameters over the Gas-solid Two-phase Flow in the 2D Riser of a Circulating Fluidized Bed", Powder Technology, Vol.132, No. 2-3, pp. 216-225.

Cabezas-Gómez, L. and Milioli, F.E., 2001, "Gas-solids two-phases flow in the riser of circulating fluidized bed: mathematical modelling and numerical simulation", RBCM-J. of the Brazilian Soc. Mechanical Sciences, Vol. 33, No. 2, pp. 179-200.

Celmiňš, A., 1988, "Representation of two-phase flows by volume averaging", International Journal of Multiphase Flow, Vol.14, No. 1, pp. 8190.

Chapman, S. and Cowling, T.G., 1970, "The mathematical theory of non-uniform gases", 3rd ed., Cambridge University Press, Cambridge, U.K.

Ding, J. and Gidaspow, D., 1990, "A bubbling model using kinetic theory of granular flow", AIChE Journal, Vol.36, No. 4, pp. 523-538.

Enwald, H., Peirano, E. and Almstedt, A.-E., 1996, "Eulerian two-phase flow theory applied to fluidization", International Journal of Multiphase Flow, Vol.22, Supplement, pp. 21-66.

Ergun, S., 1952, "Fluid flow through packed columns", Chemical Engineering Progress, Vol.48, No. 2, pp. 89-94.

Fan, L.S. and Zhu, C., 1998, "Principles of gas-solid flows", Cambridge University Press, U.K.

Gidaspow, D., Tsuo, Y.P. and Luo, K.M., 1989, "Computed and Experimental Cluster Formation and Velocity Profiles in Circulating Fluidized Beds", In Grace, J.R., Shemilt, L.W. and Bergougnou, M.A., Fluidization, vol. VI, p. 81, Engineering Foundation, New York.

Gidaspow, D., 2000, "Computation and measurement of structure and turbulence in risers and bubbling beds", Multiphase Fluid Dynamics Research Consortium, Semi-Annual Meeting and Review, Apr. 12-14, Albuquerque, New Mexico.
Gidaspow, D., 1994, "Multiphase flow and fluidization: continuum and kinetic theory descriptions", Academic Press Inc, Bostom.

Gidaspow, D. and Huilin, H.L., 1998, "Equation of state and radial distribution functions of FCC particles in a CFB", AIChE Journal, Vol.44, No. 2, pp. 279-293.

Jayaswal, U., 1991, "Hydrodynamics of multiphase flows: separation, dissemination and fluidization", Ph.D. Thesis, Illinois Institute of Technology, Chicago.

Luo, K.M., 1987, "Dilute, Dense-Phase and Maximum Solids-Gas Transport", Ph.D. Thesis, Illinois Institute of Technology, Chicago.

Matonis, D., Gidaspow, D. and Bahary, M., 2001, "CFD simulation of flow and turbulence in a slurry bubble column", AIChE Journal, Vol.48, No. 7, pp. 1413-1429.

Mudde R.F., Lee D.J., Reese J. and Fan, L.-S., 1997, "Role of coherent structures on Reynolds stresses in a 2-D bubble column", AICHE Journal, Vol.43, No. 4, pp. 913-926.

Neri, A. and Gidaspow, D., 2000, "Riser hydrodynamic: simulation using kinetic theory", AIChE Journal, Vol.46, No. 1, pp. 52-67.

Pan, Y., Dudukovic, M.P. and Chang, M., 2000, "Numerical investigation of gas-driven flow in 2-D bubble columns", AIChE Journal, Vol.46, No. 3, pp. 434-449.

Peirano, E. and Leckner, B., 1998, "Fundamentals of turbulent gas-solid flows applied to circulating fluidized bed combustion", Progress in Energy and Combustion Science, Vol.24, No. 4, pp. 259-296.

Sinclair, J.L., 1997, Hydrodynamic Modeling, In Grace, J.R., Avidan, A.A. and Knowlton, T.M., "Circulating Fluidized Beds", p. 149, Chapman \& Hall.

Sundaresan, S., 2000, "Modeling the hydrodynamics of multiphase flow reactors: Current status and challenges", AIChE Journal, Vol.46, No. 6, pp. 1102-1105.

Tennekes, H. and Lumley, J.L., 1977, "A first course in turbulence", MIT Press, Cambridge, MA.

Tsuo, Y.P. and Gidaspow, D., 1990, "Computation of flow patterns in circulating fluidized beds", AIChE Journal, Vol.36, No. 6, pp. 885-896.

Wen, C.Y. and Yu, Y.H., 1966, "Mechanics of fluidization", Chemical Engineering Progress Symposium Series, Vol.62, No. 62, pp. 100-111. 